\title{
The Impact of NRZ Data Asymmetry on the Performance of a Space Telemetry System
}

\author{
T. M. Nguyen \\ Telecommunications Systems Section
}

\begin{abstract}
The telemetry data asymmetry due to rising and falling voltage transitions can cause undesired spectral components at the output of a spacecraft transmitter. The performance of a space telemetry system can potentially degrade because of these undesired components. This article derives an expression for the power spectral density of an asymmetric nonreturn-to-zero (NRZ) data stream. This formula is then used to investigate how the data bandwidth varies with data asymmetry.
\end{abstract}

At the receiver end, the threshold levels of undesired spectral components that fall into the carrier-tracking-loop bandwidth are determined by examining the derived spectral density. Further, based on this formula, a simple technique is developed for the computation of bit signal-to-noise ratio (SNR) degradation due to data asymmetry. The telemetry bit $S N R$ degradations derived using this technique are compared with results obtained previously and with measurements by the Electronic Systems Test Laboratory (ESTL) at the Lyndon B. Johnson Space Center.

\section{Introduction}

The performance of a space telemetry link will be degraded by data asymmetry because of non-ideal rising and falling voltage transitions. The data asymmetry can cause undesired spectral components that fall within the carriertracking-loop bandwidth, causing potential interference to the carrier tracking. In addition, these undesirable spectral components can cause telemetry-bit signal-to-noise ratio degradation. Space telemetry links often operate under imposed bandwidth constraints. When two adjacent data channels overlap in the frequency domain, the per- formance of a space telemetry system can be degraded severely. With these concerns in mind, a communications engineer is interested in determining the power spectral density of the transmitted data.

The Consultative Committee for Space Data Systems (CCSDS) recommends the binary NRZ format for spacecraft-to-ground telemetry data [1]. The transmitted binary NRZ data stream considered in this article is asymmetric in time with a transition density, $p_{t}$, which is less than or equal to $\frac{1}{2}$. The data-asymmetry model for the 
binary NRZ data used in this analysis employs the first model presented in [2] (i.e., +1 NRZ symbols are elongated by $\Delta T_{s} / 2$, relative to their nominal value of $T_{s}$ seconds, when a negative-going data transition occurs; -1 symbols are shortened by the same amount when a positivegoing data transition occurs; and the symbols maintain their nominal $T_{3}$ seconds when no transitions occur). This article derives an expression for the power spectral density of an asymmetric NRZ data stream. Using this expression, the data bandwidth can be measured as a function of the data asymmetry for a specified transition density.

Based on the derived formula for the power spectral density, this article determines the levels of undesired spectral components caused by the data asymmetry that falls into the carrier-tracking-loop bandwidth and the amount of telemetry SNR degradation as a function of the amount of data asymmetry. The threshold level of the undesired spectral components causing potential interference to the carrier tracking is characterized by the interference-tocarrier power ratio $(I / C)$. A general expression for the $I / C$ as a function of the amount of data asymmetry is derived.

In 1978, Simon et al. investigated the effects of data asymmetry on system performance [2]. Using this technique, the bit SNR degradations were calculated by evaluating the average bit error rate (BER) in the presence of data asymmetry and then computing the additional bit SNR required because of asymmetry to produce the same value of BER when there is no data asymmetry.

This article develops a simple technique to determine the amount of telemetry-bit SNR performance degradation as a function of the amount of data asymmetry for a specified transition density. The technique presented here uses an approach entirely different from that presented in [2]. The results obtained by this technique are then compared with the results presented in [2].

This article begins with the derivation of the power spectral density for an asymmetric NRZ data stream generated by a purely random source. That is followed by an investigation of the effect of the data asymmetry on the data bandwidth. Next, an analysis of the effect of data asymmetry on the performance of the carrier tracking loop is given along with a set of curves used to determine the critical amount of data asymmetry that produces undesired spectral components, which cause harmful interference to carrier tracking. Finally, this article develops a simple technique to assess the telemetry-bit SNR degradation caused by various values of data asymmetry and then compares the results obtained from this technique with the results presented in [2], and the test results taken in the Electronic Systems Test Laboratory (ESTL).

\section{Derivation of the Asymmetric NRZ Data Power Spectrum}

Consider a random quaternary source that every $T_{s}$ seconds transmits a signal from the set $\left\{g_{i}(t) ; i=1,2,3,4\right\}$ with probability $p_{i}$. In the following analysis, the signal is assumed to be generated by a purely random source where the signal transmitted in a given signaling interval is independent of those transmitted in previous signaling intervals. If one defines $G_{i}(f)$ as the Fourier transforms of the symbols $g_{i}(t)$, for $i=1,2,3$, and 4 , respectively, then the power spectral density of the random data stream generated by this source is given by [3]

$$
\begin{aligned}
& S(f)=R_{s}^{2} \mid p_{1} G_{1}(0)+p_{2} G_{2}(0)+p_{3} G_{3}(0) \\
& +\left.p_{4} G_{4}(0)\right|^{2} \delta(f)+2 R_{s}^{2} \sum_{m=1}^{\infty} \mid p_{1} G_{1}\left(m R_{s}\right)+p_{2} G_{2}\left(m R_{s}\right) \\
& +p_{3} G_{3}\left(m R_{s}\right)+\left.p_{4} G_{4}\left(m R_{s}\right)\right|^{2} \delta\left(f-m R_{s}\right) \\
& +R_{s}\left\{p_{1}\left(1-p_{1}\right)\left|G_{1}(f)\right|^{2}+p_{2}\left(1-p_{2}\right)\left|G_{2}(f)\right|^{2}\right. \\
& \left.+p_{3}\left(1-p_{3}\right)\left|G_{3}(f)\right|^{2}+p_{4}\left(1-p_{4}\right)\left|G_{4}(f)\right|^{2}\right\} \\
& -2 R_{s}\left\{p_{1} p_{2} \Re\left[G_{1}(f) G_{2}^{*}(f)\right]+p_{1} p_{3} \Re\left[G_{1}(f) G_{3}^{*}(f)\right]\right. \\
& +p_{1} p_{4} \Re\left[G_{1}(f) G_{4}^{*}(f)\right]+p_{2} p_{3} \Re\left[G_{2}(f) G_{3}^{*}(f)\right] \\
& \left.+p_{2} p_{4} \Re\left[G_{2}(f) G_{4}^{*}(f)\right]+p_{3} p_{4} \Re\left[G_{3}(f) G_{4}^{*}(f)\right]\right\}
\end{aligned}
$$

Ilere, $R_{s}=1 / T_{s}$ is the symbol rate (or signaling frequency), the asterisk denotes a complex conjugate, and $\Re[\cdot]$ denotes the real part.

It is assumed that the first data-asymmetry model presented in [2] (see Fig. 1) is used in the following analysis. For this particular asymmetric NRZ data stream,

$$
\begin{aligned}
& g_{1}(t)= \begin{cases}+A & -\left(T_{s} / 2\right)<t \leq\left(T_{s} / 2\right)(1+\Delta) \\
0 & \text { elsewhere }\end{cases} \\
& g_{2}(t)= \begin{cases}-A & -\left(T_{s} / 2\right)<t \leq\left(T_{s} / 2\right)(1-\Delta) \\
0 & \text { elsewhere }\end{cases}
\end{aligned}
$$




$$
\begin{aligned}
& g_{3}(t)= \begin{cases}+A & -\left(T_{s} / 2\right)<t \leq\left(T_{s} / 2\right) \\
0 & \text { elsewhere }\end{cases} \\
& g_{4}(t)= \begin{cases}-A & -\left(T_{s} / 2\right)<t \leq\left(T_{s} / 2\right) \\
0 & \text { elsewhere }\end{cases}
\end{aligned}
$$

The Fourier transforms $G_{1}(f), G_{2}(f), G_{3}(f)$, and $G_{4}(f)$ of the symbols $g_{1}(t), g_{2}(t), g_{3}(t)$, and $g_{4}(t)$, respectively, can be shown to have the following forms:

$$
\begin{aligned}
& G_{1}(f)=\frac{A e^{-\left(j \pi f T_{s} \Delta\right) / 2}}{\pi f} \sin \left(\pi f T_{s}(1+\Delta / 2)\right) \\
& G_{2}(f)=-\frac{A e^{\left(j \pi f T_{\bullet} \Delta\right) / 2}}{\pi f} \sin \left(\pi f T_{s}(1-\Delta / 2)\right) \\
& G_{3}(f)=\frac{A \sin \left(\pi f T_{s}\right)}{\pi f} \\
& G_{4}(f)=-G_{3}(f)
\end{aligned}
$$

From Eqs. (6), (7), (8), and (9),

$$
\begin{aligned}
& G_{1}(0)=\lim _{f \rightarrow 0} G_{1}(f)=A T_{s}(1+\Delta / 2) \\
& G_{2}(0)=\lim _{f \rightarrow 0} G_{2}(f)=-A T_{s}(1-\Delta / 2) \\
& G_{3}(0)=-G_{4}(0)=\lim _{f \rightarrow 0} G_{3}(f)=A T_{s}
\end{aligned}
$$

If $p$ is the probability of transmitting a positive pulse, i.e., $p=\operatorname{Pr}\left\{g_{i}(t)=+A\right\}$, and $p_{t}$ the transition density, i.e., $p_{t}=\operatorname{Pr}\left\{g_{i}(t) \neq g_{i+1}(t)\right\}$, then $p_{i}$, for $i=1,2,3$, and 4 is given by

$$
\begin{aligned}
& p_{1}=\operatorname{Pr}\left\{g_{i}(t)=g_{1}(t)\right\}=p p_{t} \\
& p_{2}=\operatorname{Pr}\left\{g_{i}(t)=g_{2}(t)\right\}=(1-p) p_{t} \\
& p_{3}=\operatorname{Pr}\left\{g_{i}(t)=g_{3}(t)\right\}=p\left(1-p_{t}\right) \\
& p_{4}=\operatorname{Pr}\left\{g_{i}(t)=g_{4}(t)\right\}=(1-p)\left(1-p_{t}\right)
\end{aligned}
$$

For a purely random source, the transition density $p_{t}$ can easily be shown to be

$$
p_{t}=2 p(1-p)
$$

Inserting the terms from Eqs. (6) through (16) into Eq. (1) and carrying out the necessary mathematics shows the power spectral density $S_{d}(f)$ for the asymmetric NRZ data stream generated by a purely random source to be

$$
\begin{aligned}
S_{d}(f)= & \left(A^{2} T_{s}\right) \frac{\sin ^{2}\left(\pi f T_{s}\right)}{\left(\pi f T_{s}\right)^{2}}\left[a_{1}\left(p_{t}\right)+a_{2}\left(p, p_{t}, \eta\right)\right] \\
& +\underbrace{\left(A^{2} T_{s}\right) a_{3}\left(p_{t}, \eta\right) \frac{\sin ^{2}\left(\pi f T_{s} \eta\right)^{2}}{\left(\pi f T_{s}\right)}}_{\text {continuous spectrum, } s_{c}(f)} \\
& +\underbrace{\left(A^{2} T_{s}\right) \frac{\sin \left(\pi f T_{s}\right)}{\left(\pi f T_{s}\right)^{2}}\left[a_{4}\left(p, p_{t}, \eta\right)-a_{5}\left(p, p_{t}\right)\right]}_{\text {dc component, } s_{d c}(f)} \\
& +\underbrace{\frac{A^{2}\left[2 p-\left(1-\eta p_{t}\right)\right]^{2} \delta(f)}{\pi^{2} p_{t}^{2}} \sum_{m=1}^{\infty} \frac{1}{m^{2}} C(m, p, \eta) \delta\left(f-m R_{s}\right)}_{\text {harmonics, } S_{h}(f)}
\end{aligned}
$$

where

$$
a_{1}\left(p_{t}\right)=p_{t}\left(1-p_{t}\right)\left[1+2\left(1-p_{t}\right)\right]-p_{t}^{3}
$$

$$
\begin{aligned}
a_{2}\left(p, p_{t}, \eta\right)= & {\left[3 p_{t}^{3}+p_{t}\left(1-p_{t}\right)[1+2(1-2 p)]\right] } \\
& \times \cos ^{2}\left(\pi f T_{s} \eta\right) \\
a_{3}\left(p_{t}, \eta\right)= & p_{t}\left(1+p_{t}^{2}-p_{t}\right) \cos ^{2}\left(\pi f T_{s}\right) \\
& +p_{t}^{3} \cos \left(2 \pi f T_{s} \eta\right) \\
a_{4}\left(p, p_{t}, \eta\right)= & p_{t}\left(1-p_{t}\right)(1-2 p)\left[0.5 \cos \left(2 \pi f T_{s} \eta\right)\right. \\
& \left.-p \sin \left(2 \pi f T_{s} \eta\right)\right]
\end{aligned}
$$




$$
\begin{gathered}
a_{5}\left(p, p_{t}\right)=0.5 p_{t}\left(1-p_{t}\right)(1-2 p) \\
C(m, p, \eta)=\sin ^{2}(m \pi \eta)\left[\cos ^{2}(m \pi \eta)-(1-2 p)^{2} \sin ^{2}(m \pi \eta)\right]
\end{gathered}
$$

$$
\eta=\frac{\Delta}{2}
$$

Equation (18) shows that the spectrum of the asymmetric NRZ data stream is a function of the probability of occurrence $p$ of the positive symbol, the transition density $p_{t}$, and the amount of data asymmetry $\eta$. However, the power spectral density can be expressed in terms of the transition density by solving Eq. (17) for $p$ and substituting the result in Eq. (18). Note that Eq. (18) is valid only for $p_{t} \leq 1 / 2$.

It is also interesting to evaluate the power spectral density $S_{d}(f)$ for the case of random data with equiprobable symbols, i.e., $p=p_{t}=1 / 2$. For this special case, from Eq. (19) through (23),

$$
\begin{aligned}
a_{1}(1 / 2)= & 3 / 8 \\
a_{2}(1 / 2,1 / 2, \eta)= & (5 / 8) \cos ^{2}\left(\pi f T_{s} \eta\right) \\
a_{3}(1 / 2,1 / 2, \eta)= & (3 / 8) \cos ^{2}\left(\pi f T_{s}\right) \\
& +(1 / 8) \cos ^{2}\left(\pi f T_{3} \eta\right) \\
a_{4}(1 / 2,1 / 2, \eta)= & a_{5}(1 / 2,1 / 2)=0
\end{aligned}
$$

Where the terms from Eqs. (26) through (29) are substituted into Eq. (18), the power spectral density for the asymmetric NRZ random data stream with equiprobable symbols is

$$
\begin{aligned}
& S_{d}^{o}(f)=\left(A^{2} T_{s} / 8\right)\left[\frac{\sin ^{2}\left(\pi f T_{s}\right)}{\left(\pi f T_{s}\right)^{2}}\right]\left[3+5 \cos ^{2}\left(\pi f T_{s} \eta\right)\right] \\
& +\underbrace{\left(A^{2} T_{s} / 8\right)\left[\frac{\sin ^{2}\left(2 \pi f T_{s} \eta\right)}{\left(\pi f T_{s}\right)^{2}}\right]\left[3 \cos ^{2}\left(\pi f T_{s}\right)+\cos ^{2}\left(2 \pi f T_{s} \eta\right)\right]}_{\text {continuous spectrum, } S_{c}^{o}(f)} \\
& +\underbrace{A^{2}(0.5 \eta)^{2} \delta(f)}_{\text {dc component, } S_{d c}^{o}(f)}
\end{aligned}
$$

$$
+\underbrace{\frac{A^{2}}{2 \pi^{2}} \sum_{m=1}^{\infty} \frac{1}{m^{2}} C(m, 1 / 2, \eta) \delta\left(f-m R_{s}\right)}_{\text {harmonics, } S_{h}^{\circ}(f)}
$$

Here, from Eq. (24), $C(m, 1 / 2, \eta)$ is

$$
C(m, 1 / 2, \eta)=(1 / 4) \sin ^{2}(2 m \pi \eta)
$$

The continuous spectrum $S_{c}^{o}(f)$ of Eq. (30) is plotted in Fig. 2, which illustrates the normalized data power spectra (i.e., $S_{c}^{o}(f) / E$, here $E=A^{2} T_{s}$ ) for several data asymmetry $\eta$. If $B W$ is defined as the one-sided bandwidth of the NRZ data stream, then the total data power that lies in the frequency domain $(-B W, B W)$ can be determined. Figure 3 shows the functional dependence of $B W$ on the data asymmetry for 90.3 percent and 89 percent of the total data power containment. As expected, for a fixed data rate, the one-sided bandwidth $B W$ increases as $\eta$, the data asymmetry, increases.

\section{The Effects of Data Asymmetry on the Carrier Tracking Loop}

The space telemetry signal recommended by the CCSDS can be expressed mathematically as [1]

$$
s_{T}(t)=\sqrt{2} A_{T} \sin \left[\omega_{c} t+m_{T} d(t) P(t)\right]
$$

where $A_{T}$ is the transmitted rms voltage, $\omega_{c}=2 \pi f_{c}$ is the angular carrier center frequency in rads/sec, $m_{T}$ is the telemetry modulation index in rads, $d(t)$ is the data waveform generated by the binary $( \pm 1)$ NRZ data sequence, and $P(t)$ is the subcarrier waveform.

The most interesting subcarrier waveforms in space applications are square-wave for deep-space missions (Category B) and sine-wave for near-Earth missions (Category A) [1]. For example, the Category $B$ subcarrier waveform can be represented by the Fourier series expansion

$$
P(t)=\frac{4}{\pi} \sum_{k=1}^{\infty} \frac{\cos \left[(2 k-1) 2 \pi f_{s c} t\right]}{2 k-1}
$$

Here, $f_{s c}$ is the subcarrier frequency in $\mathrm{Hz}$.

If the data sequence $d(t)$ is asymmetric as shown in Fig. 1 (with equiprobable symbols and $A=1$ ), then the power spectral density $S_{d}(f)$ of this asymmetric NRZ data stream is found in Eq. (18). From Eqs. (32) and (33), it 
is straightforward to show that the power spectral density of the deep-space telemetry signal is equal to

$$
\begin{aligned}
& S_{T}(f)=A_{T}^{2} \cos ^{2}\left(m_{T}\right) \delta\left(f-f_{c}\right) \\
& +\frac{4}{\pi^{2}} A_{T}^{2} \sin ^{2}\left(m_{T}\right) \\
& \times \sum_{k=1}^{\infty}\left[\frac{S_{d}\left(f-f_{c}-(2 k-1) f_{s c}\right)+S_{d}\left(f-f_{c}+(2 k-1) f_{s c}\right)}{(2 k-1)^{2}}\right]
\end{aligned}
$$

Here, $S_{d}(f)$ is given in Eq. (18) with $A=1$.

The index value $k$, see Eq. (34), denotes the $k$ th harmonic due to the subcarrier, and $m$, see Eq. (18), denotes the $m$ th harmonic due to data asymmetry. When mixing the $m$ th harmonic with the $k$ th harmonic, a "third harmonic" is produced. This third harmonic can have a spectral component that falls into the carrier-tracking-loop bandwidth. It is desirable to locate this third harmonic by examining all possible combinations of the index values $k$ and $m$. From Eqs. (18) and (34), it is easy to show that when

$$
m=2 n k-n
$$

for $k$ and $n$ are integers. The harmonics $S_{h}(f)$, from Eq. (18), when mixed with the subcarrier signal, will produce spectral components that fall on the top of the carrier spectral component. Here, $n$ is the ratio (assumed to be an integer) of the subcarrier frequency-to-bit rate, i.e., $n=f_{s c} / R_{s}$. The number of subcarrier cycles per symbol is chosen so that the interaction between data sidebands and the residual $\mathrm{RF}$ carrier is minimum. In practice, $f_{s c}$ is usually chosen to be three times the data rate, i.e., $n=3$.

The plot of Eq. (35) is shown in Fig. 4, which illustrates the functional behavior of the $k$ th harmonic due to subcarrier versus $m$ th harmonic due to data asymmetry. From this figure, for a given $k$, one can determine the corresponding $m$ th harmonic that, when mixed with the $k$ th harmonic, will produce a spectral component that falls on the top of the carrier spectral component causing potential interference to the carrier tracking loop. Knowing the values of $m$ and $k$, the interference-to-carrier power ratio $I / C$ can be calculated. From Eqs. (18) and (34), it is easy to show that

$$
I / C=\frac{8 p_{t}^{2} \tan ^{2}\left(m_{T}\right) C(m, p, \eta)}{\pi^{4} m^{2}(2 k-1)^{2}}
$$

For $m=2 n k-n, k$ and $n$ are integers.

$$
\text { For } p=p_{t}=1 / 2 \text {, Eq. (34) becomes }
$$

$$
I / C=\frac{4 \tan ^{2}\left(m_{T}\right) C(m, 1 / 2, \eta)}{\pi^{4} m^{2}(2 k-1)^{2}}
$$

For $m=2 n k-n, k$ and $n$ are integers, and where $C(m, 1 / 2, \eta)$ is given in Eq. (31).

The plot of Eq. (37) is shown in Figs. 5 and 6 for various values of $n, k$, and $m$. As expected, Fig. 5 shows that the $I / C$ increases as the telemetry modulation index increases. This occurs because less power is allocated to the carrier component as the telemetry modulation index increases. Furthermore, Fig. 5 shows that the $I / C$ decreases as both $k$ and $m$ increase. Figure 6 shows that the $I / C$ decreases as $n$, the subcarrier frequency-to-bit rate, increases.

Figures 5 and 6 can be used to determine the critical amount of data asymmetry that will produce the threshold level of $I / C$. As an example, from Fig. 5, for $m_{T}=1.35$ rads, $n=3, k=1$, and $m=3$, one finds that $I / C=-19.46 \mathrm{~dB}$ for $\eta=8.27$ percent. However, for a deep-space mission, the maximum allowable $C W$ interference for the carrier tracking is $(I / C)_{\max }=-15 \mathrm{~dB}$ [5]. Thus, for this particular case, the undesired spectral component generated by data asymmetry does not exceed the maximum allowable interference when the data asymmetry $\eta$ is equal to 8.27 percent. Figure 5 shows that, for $m_{T}=1.35 \mathrm{rads}, 0.95 \mathrm{rads}, n=3, k=1,2$, and $m=3,5$, the interference-to-carrier power ratio does not exceed the maximum allowable interference, i.e., $I / C<(I / C)_{\max }$, for any value of $\eta$.

\section{Telemetry-Bit SNR Degradation Due to Data Asymmetry}

To quantitatively determine the telemetry-bit SNR degradation due to data asymmetry, one must define all the degradation sources created by the data asymmetry. In this section, it is assumed that the telemetry BER degradation due to imperfect bit synchronization is negligible (only on the order of a few tenths of a dB) [2]. Furthermore, the telemetry BER degradation due to imperfect subcarrier tracking is also ignored. This is the case because the subcarrier frequency is usually much higher than the data symbol rate; hence, the subcarrier tracking loop will not be affected by the data asymmetry. This is particularly true for a specific implementation of the subcarrier tracking loop described in [4]. Thus, there remain three major 
sources of degradation that the data asymmetry created, namely:

(1) Undesired discrete spectral components

(2) Continuous spectral distortion

(3) Matched filtering loss

The undesired discrete spectral components created by data asymmetry can cause potential interference to the telemetry data channel. If one denotes $\Delta_{u}$ as the bit SNR degradation due to the presence of the undesired spectral components, then it is straightforward to show that

$$
\Delta_{u}=10 \log _{10}\left[1+\frac{1}{N_{0}(B W)} \int_{0}^{B W} S_{I}(f) d f\right]
$$

where $N_{0}$ is the one-sided noise spectrum density, $B W$ is the one-sided data bandwidth, and $S_{I}(f)$ is the interference power spectrum density.

The above equation assumes that the total noise spectral density seen by the telemetry data channel is the thermal noise plus the interference spectral density. If the onesided bandwidth is equal to the bit rate, i.e., $B W=R_{a}$, then, from Eq. (18),

$$
S_{I}(f)=S_{d c}(f)+\frac{2 A^{2} P_{t}^{2}}{\pi^{2}} C(1, p, \eta) \delta\left(f-R_{s}\right)
$$

where $S_{d c}(f)$ is defined in Eq. $(18)$, and $C_{t}(1, p, \eta)$ is given in Eq. (24) with $m=1$.

For $p=P_{t}=1 / 2, S_{d c}(f)=S_{d c}^{o}(f)$, see Eq. (30). Thus, from Eq. (30), Eq. (39) can be rewritten

$$
S_{I}(f)=A^{2}(0.5 \eta)^{2} \delta(f)+\frac{A^{2}}{2 \pi^{2}} C(1,1 / 2, \eta) \delta\left(f-R_{s}\right)
$$

In this case, $C(1,1 / 2, \eta)=(1 / 4) \sin ^{2}(2 \pi \eta)$ is given in Eq. (31) with $m=1$.

Therefore, for $p=p_{t}=1 / 2$, from Eq. (40), Eq. (38) can be written as

$$
\Delta_{u}=10 \log _{10}\left[1+\frac{E_{b}}{N_{0}}\left((0.5 \eta)^{2}+\frac{C(1,1 / 2, \eta)}{2 \pi^{2}}\right)\right]
$$

Here, $E_{b}=A^{2} T_{s}=$ bit energy. As an example for Category $\mathrm{B}$, the bit energy is found to be: $E_{b}=\left(A_{T}^{2} T_{s}\right)$ $\sin ^{2}\left(m_{T}\right)$, see Eq. (34).
Equation (41) assumes that all the harmonics with frequency components greater than $R_{s}$, i.e., $f>R_{s}$, have been filtered out by the predetection filter (assumed to be of the brick-wall type).

Figure 2 shows that the continuous component of the telemetry spectral density is distorted by data asymmetry. Spectral distortion can cause further degradation in bit SNR. If $\Delta_{d}$ is the bit SNR degradation due to spectral distortion, then $\Delta_{d}$ can be defined as

$$
\Delta_{d}=10 \log _{10}\left[\frac{a_{1}}{a_{2}}\right]
$$

where

$$
a_{1}=\int_{-R_{e}}^{R,} S_{c n}(f) d f
$$

Here, $S_{c n}(f)=S_{c}(f) / E_{b}$, with $S_{c}(f)$ as defined in Eq. (18). For $p=p_{t}=1 / 2, S_{c}(f)=S_{c}^{o}(f)$. Here, $S_{c}^{o}(f)$ is given in Eq. (30). Then

$$
a_{2}=\int_{-R_{1}}^{R,} S_{u d}(f) d f
$$

Here, $S_{u d}(f)$ is the undistorted normalized (by $E_{b}$ ) spectral density, which is given by [3]

$$
S_{u d}(f)=\left[\frac{\sin \left(\pi f / R_{s}\right)}{\left(\pi f / R_{s}\right)}\right]^{2}
$$

It is well known that for binary NRZ data signals, the matched filter is an integrate and dump filter. Since the binary NRZ data stream is distorted by the rising and falling voltage transitions, the integrate and dump filter is no longer the matched filter for detecting the binary NRZ data signal. If $\Delta_{m}$ is the bit SNR degradation due to the matched filter, then $\Delta_{m}$ can be defined as

$$
\Delta_{m}=20 \log _{10}\left[\frac{\mu}{\mu_{0}}\right]
$$

Here the mean value of the matched filter output signal of the undistorted signal is represented by $\mu$, and the mean

value of the matched filter output signal of the distorted signal is represented by

$$
\mu_{0}=\sqrt{E_{b} T_{s}}
$$


Since the NRZ data stream is asymmetric with $p$ as the probability of occurrence of the $+A$ symbol and $(1-p)$ for the $-A$ symbol, the output of the matched filter depends on the polarity of the symbol over which it is integrating and that of the preceding and succeeding symbols. Thus, the mean value of the matched filter output can be computed by examining eight possible three-symbol sequences. From Appendix A, Fig. 2, of [6], the mean of the matched filter output signal in the presence of data asymmetry can be easily shown to have the following form:

$$
\mu=\sqrt{E_{b} T_{s}}\left\{p^{3}+(1-p)\left[p(3-2 \eta)+(1-p)^{2}\right]\right\}
$$

If Eqs. (47) and (48) are substituted into Eq. (46),

$$
\Delta_{m}=20 \log _{10}\left\{p^{3}+(1-p)\left[p(3-2 \eta)+(1-p)^{2}\right]\right\}
$$

With equiprobable symbols, i.e., $p=1 / 2$, Eq. (49) becomes

$$
\Delta_{m}=20 \log _{10}\left[\frac{1}{2}(2-\eta)\right]
$$

The telemetry-bit SNR degradation due to data asymmetry is defined as the sum of the degradations due to undesired spectral components, the spectral distortion and the matched filtering loss. From Eqs. (38), (42), and (49), the bit SNR degradation is given by

$$
\Delta=\Delta_{u}+\Delta_{d}+\Delta_{m}
$$

Here $\Delta_{u}, \Delta_{d}$, and $\Delta_{m}$ are defined in Eqs. (38), (42), and (49), respectively.

Equation (51) is used to calculate the telemetry-bit SNR degradation for the specified amount of data asymmetry with bit-error probability $\left(P_{E}^{o}\right)$ as a parameter. For $p=p_{t}=1 / 2$, the results of these calculations are given in Table 1.

A plot based on the results in Table 1 is illustrated in Fig. 7. Here the bit SNR degradation as a function of data asymmetry with probability of error $\left(P_{E}^{o}\right)$ as a parameter is illustrated. This figure clearly shows that the telemetry-bit SNR degradation increases as the data asymmetry increases. This figure also shows that, for a fixed data asymmetry, the bit SNR degradation increases as the error probability decreases.

In Figs. 8 and 9, the analytical results found in Table 1 are compared with the results presented in [2]. Figure 8 illustrates the bit SNR degradation versus data asymmetry along with the comparable results obtained from [2] corresponding to no dc restoration (direct coupling, Eq. 3 of [2]) and dc restoration by capacitive coupling (Eq. 15 of [2]). In addition, Fig. 8 also shows the test results for $B T=1.125$ taken in the ESTL [2] for the sake of comparison, where $B T$ denotes the product of channel bandwidth and nominal data period. For data asymmetry of less than 20 percent, the results obtained using the simple technique presented in this article are very close to the results obtained from Eq. 15 of [2] for a capacitively coupled matched filter.

The results presented in Table 1 are further compared in Fig. 9 with the results obtained in [2] for a specific implementation of the symbol synchronizer with dc restoration based on symbol timing (Eq. 17 of [2]). The test results taken at ESTL for $B T=1.125$ and 0.75 are also shown in this figure for the sake of comparison. Again, these results are in good agreement. However, it is to be noted that the analytical results predicted by Eq. (51) were derived for a special case where the one-sided channel bandwidth $B W$ is equal to the data bit rate $R_{s}$, i.e., $B T=1$.

\section{Conclusions}

The impact of the data asymmetry on the performance of a space telemetry system has been investigated based on the derived spectral density of an asymmetric NRZ data stream. The derived power spectrum is also used to investigate how rapidly the data bandwidth increases as the amount of data asymmetry increases. It was shown that the data bandwidth increases exponentially as the amount of data asymmetry increases. An analytical model to predict the threshold levels of undesired spectral components that fall into the carrier-tracking-loop bandwidth was derived, and a simple model to predict the telemetry-bit SNR degradation due to data asymmetry was developed. It was shown that, for a specific operating condition, the carrier tracking loop does not drop lock at any value of $\eta$, the data asymmetry, and that the simple model developed in this article can be used to accurately predict the bit SNR degradation. 


\section{Acknowledgments}

The author is indebted to Drs. M. K. Simon and F. Davarian for their invaluable comments and useful suggestions during the preliminary review of this work.

\section{References}

[1] Consultative Committee for Space Data Systems, Recommendations for Space Data System Standards, Radio Frequency and Modulation Systems, Part 1: Earth Stations and Spacecraft, CCSDS 401.0-B-1, Blue Book, National Aeronautics and Space Administration, Washington, D.C., January 1987.

[2] M. K. Simon, K. Tu, and B. II. Batson, "Effects of Data Asymmetry on Shuttle Ku-Band Communications Link Performance," IEEE Transactions on Communications, vol. COM-26, no. 11, pp. 1639-1651, November 1978.

[3] W. C. Lindsey and M. K. Simon, Telecommunication Systems Engineering, Chapter 1, Englewood Cliffs, New Jersey: Prentice-Hall, Inc., 1973.

[4] Deep Space Telecommunications Systems Engineering, Chapter 5, J. Yuen, Editor, New York: Plenum Press, 1983.

[5] International Radio Consultative Committee, "Protection Criteria and Sharing Considerations Relating to Deep Space Research," Recommendations and Reports of the CCIR, 1986, XVIth Plenary Assembly, Volume II: Space Research and Radio Astronomy, Report 685-2, pp. 278-289, Dubrovnik: CCIR, 1986.

[6] W. K. Alem, G. K. Huth, and M. K. Simon, Integrated Source and Channel Encoded Digital Communication System Design Study, Final Report (R7803-7) under Contract NAS 9-15240, Axiomatix, Marina del Rey, California, March 31, 1978. 
Table 1. Telemetry-bit SNR degradation for uncoded random NRZ data (representing the change in decibels) with $p=p_{\ell}=1 / 2$

\begin{tabular}{lllllll}
\hline & \multicolumn{7}{c}{$\eta$ percent } \\
\cline { 2 - 7 } & 2 & 5 & 10 & 15 & 20 & 25 \\
\hline $10^{-2}$ & 0.09 & 0.24 & 0.53 & 0.87 & 1.26 & 1.69 \\
$10^{-3}$ & 0.09 & 0.26 & 0.60 & 1.00 & 1.46 & 1.94 \\
$10^{-4}$ & 0.1 & 0.28 & 0.66 & 1.13 & 1.65 & 2.19 \\
$10^{-5}$ & 0.1 & 0.30 & 0.73 & 1.25 & 1.83 & 2.42 \\
$10^{-6}$ & 0.1 & 0.31 & 0.79 & 1.38 & 2.00 & 2.63 \\
\hline
\end{tabular}




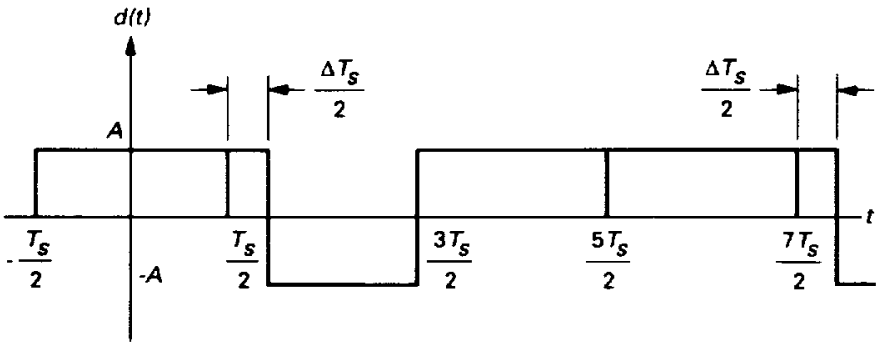

Flg. 1. Asymmetric NRZ data stream using asymmetry model 1 [2].

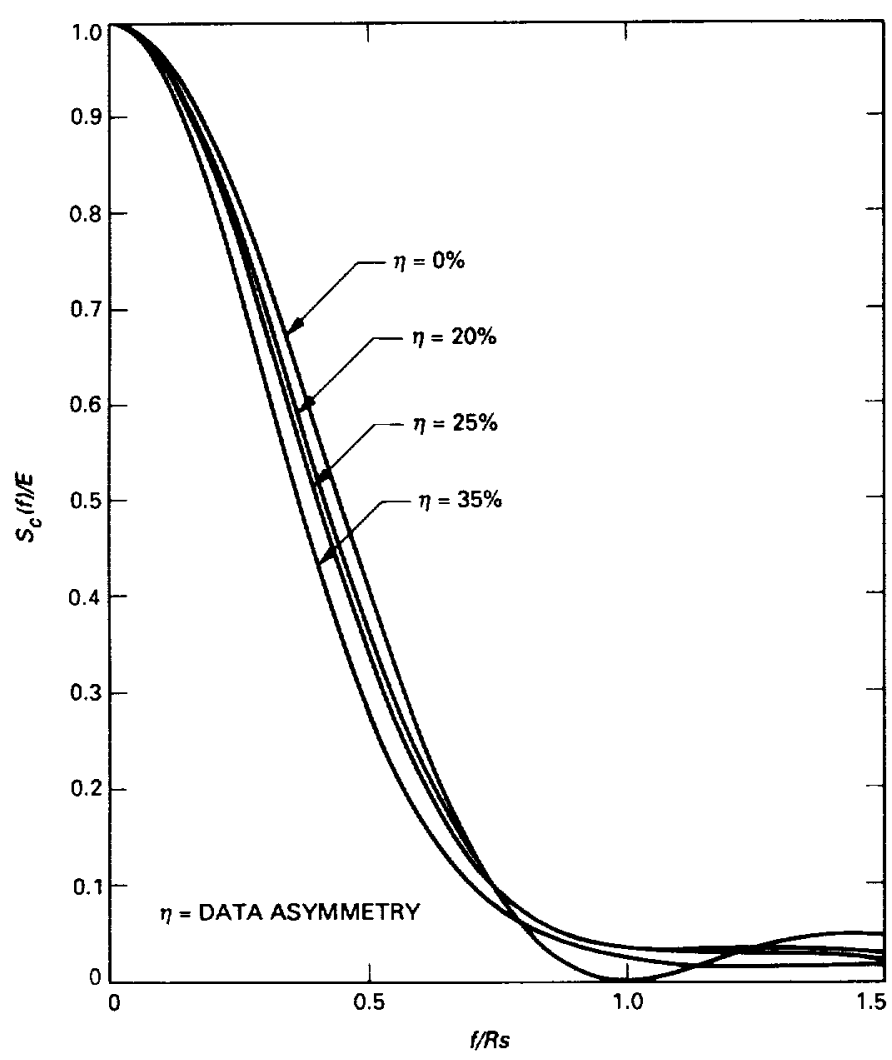

Fig. 2. Normalized power spectra versus data asymmetry.

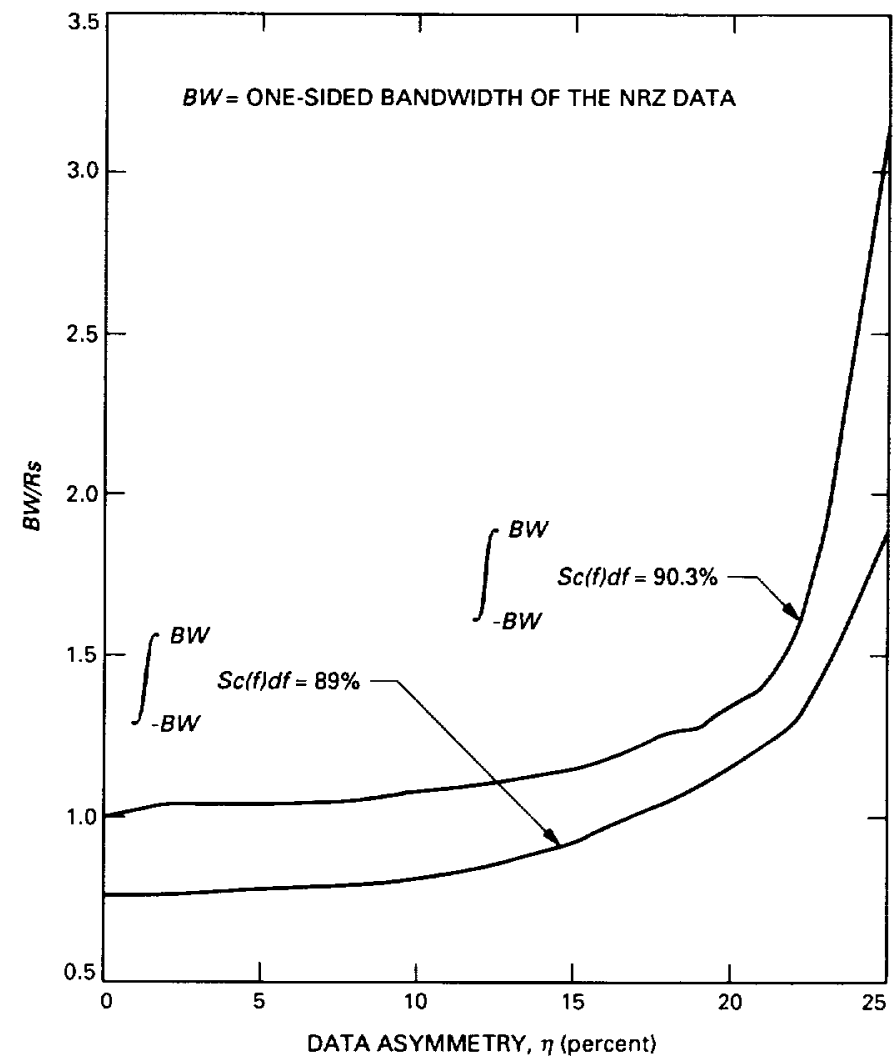

Fig. 3. One-sided bandwidth versus data asymmetry. 


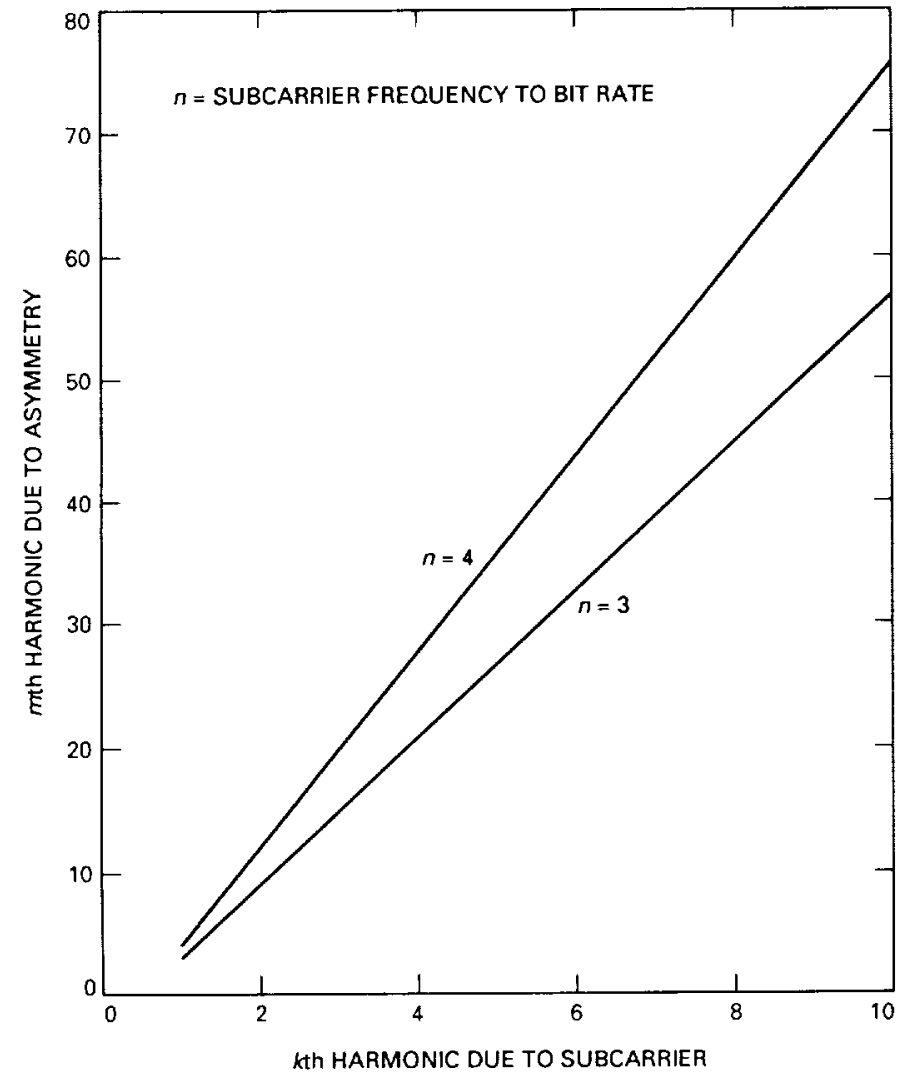

Fig. 4. Functlonal behavior of the $m$ th versus $k$ th harmonlc.

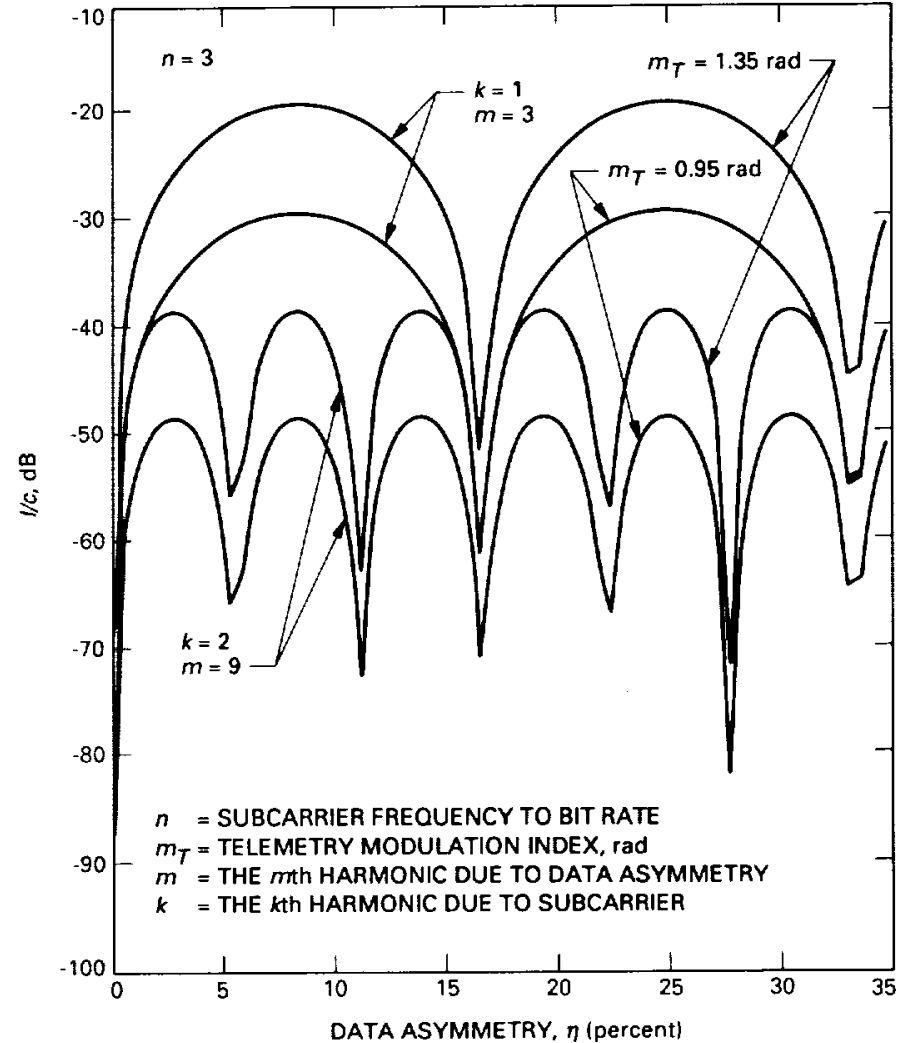

Fig. 5. Interference-to-carrler ratio versus data asymmetry for varlous values of the telemetry modulation index. 


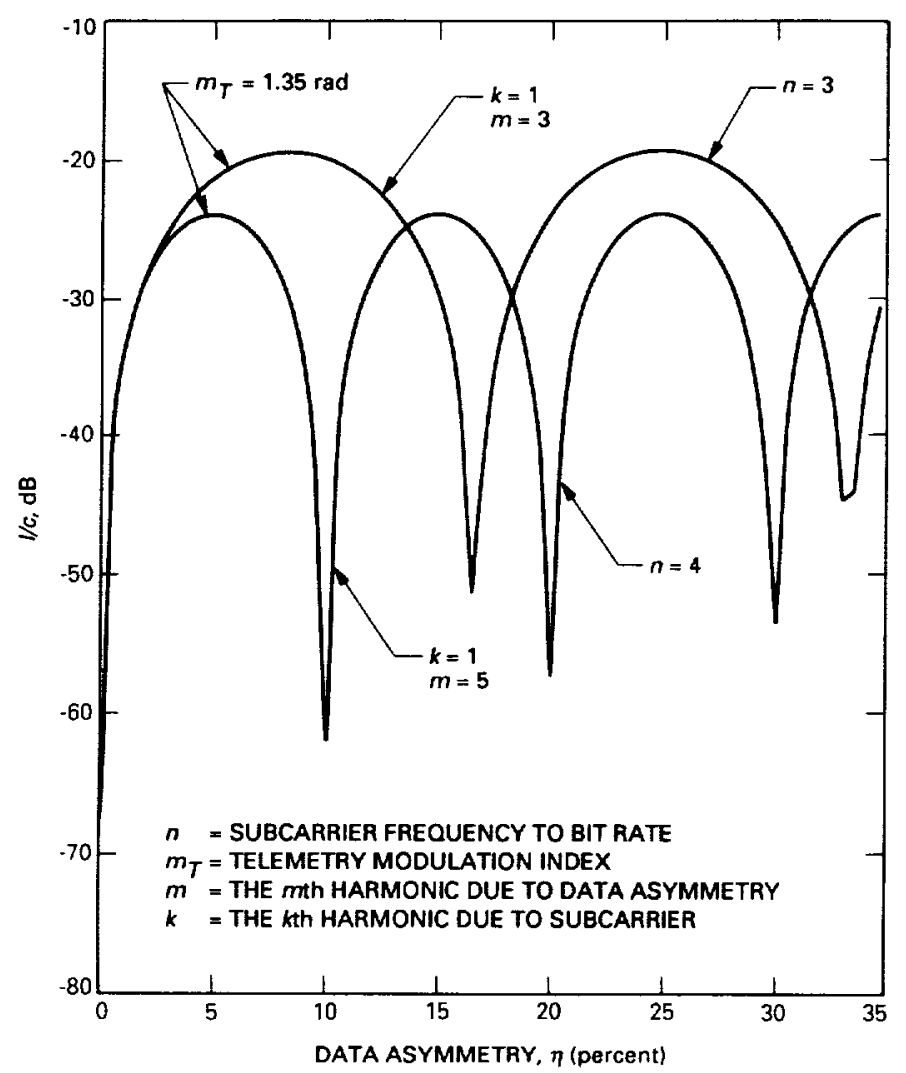

Fig. 6. Interference-to-carrier ratlo versus data asymmetry for various values of $\eta$.

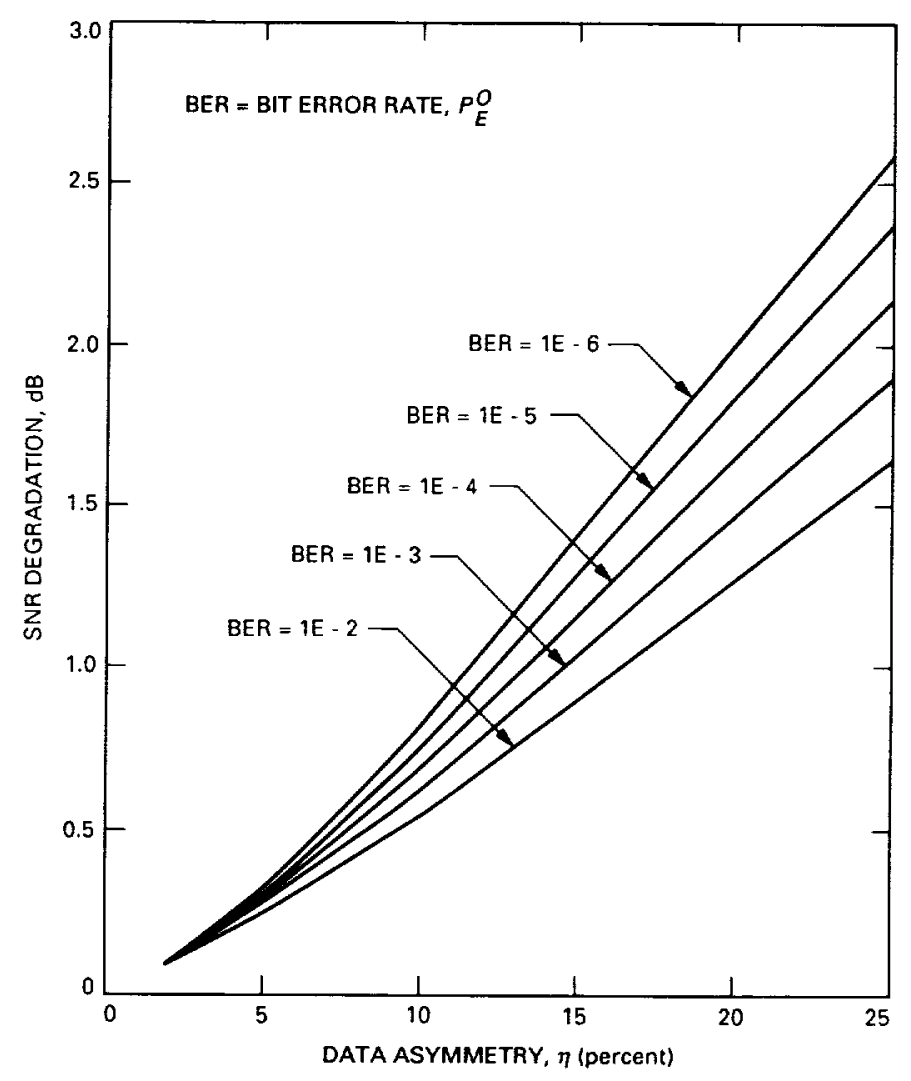

Fig. 7. Telemetry-bit SNR degradation versus data asymmetry. 


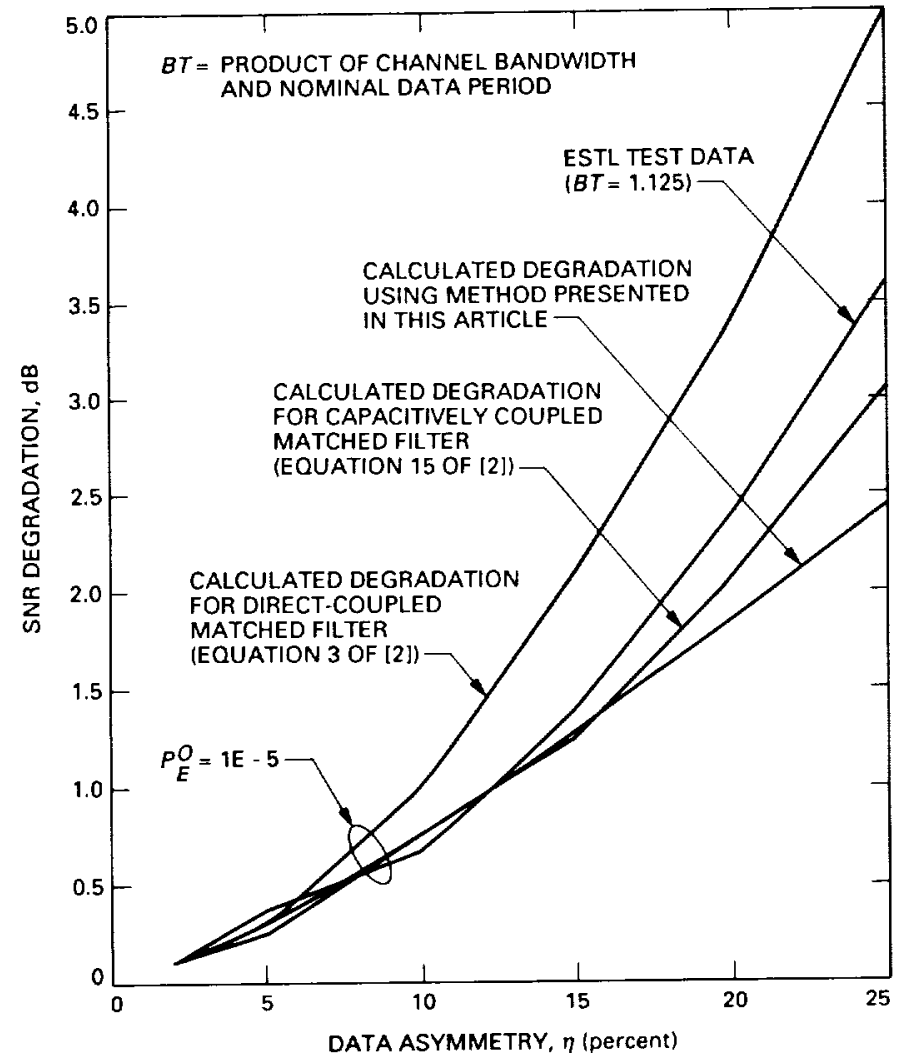

Fig. 8. Performance degradation comparison with ESTL test data for $B T=1.125$.

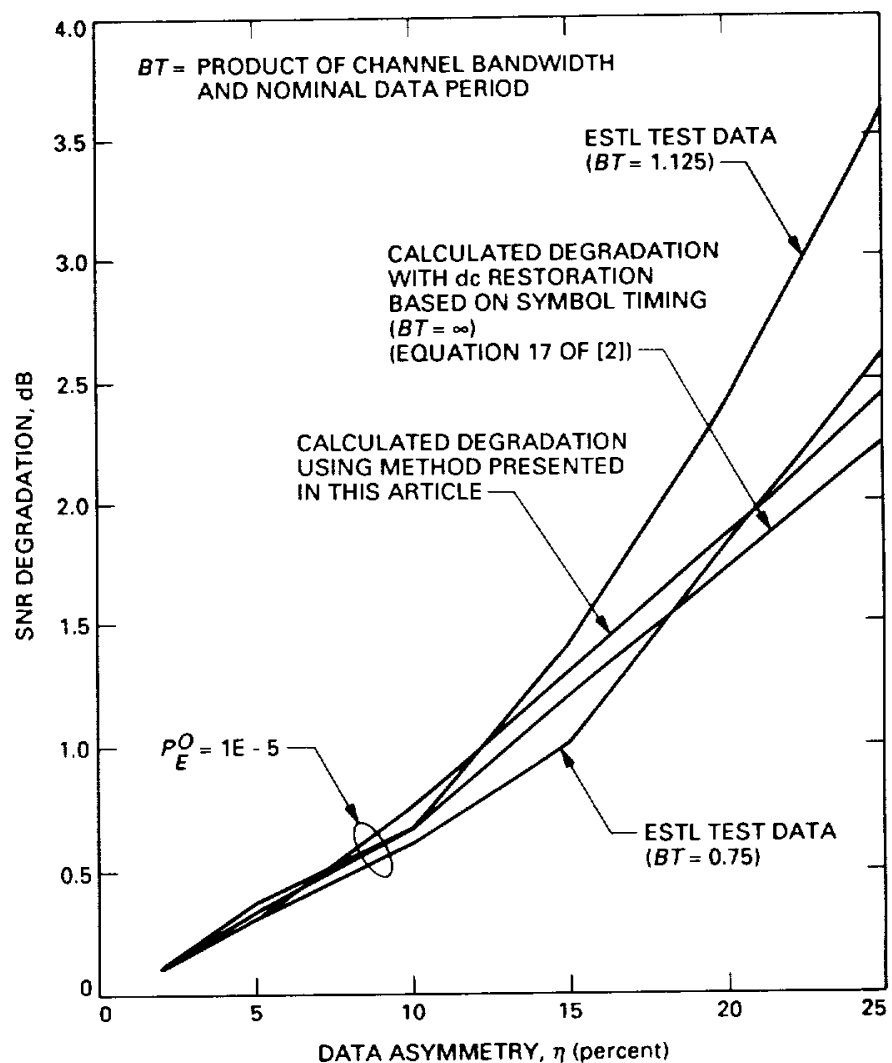

Fig. 9. Performance degradation comparison for $B T=1.125$ and $B T=0.75$. 